\title{
Reconstructing the Optical Thickness from Hoffman Modulation Contrast Images
}

\author{
Niels Holm Olsen ${ }^{124}$, Jon Sporring ${ }^{1}$, Mads Nielsen ${ }^{2}$, \\ Christina Hnida ${ }^{34}$, and Søren Ziebe ${ }^{3}$ \\ 1 3D-Lab, School of Dentistry, University of Copenhagen \\ Nørre Alle 20, 2200 Copenhagen, Denmark \\ ${ }^{2}$ IT University of Copenhagen \\ Glentevej 67, 2400 Copenhagen, Denmark \\ 3 Fertility Clinic, University Hospital \\ Blegdamsvej 9, 2100 Copenhagen, Denmark \\ 4 IH-Medical, Image House A/S \\ Store Kannikestræde 7, 1169 Copenhagen, Denmark
}

\begin{abstract}
Hoffman microscopy imaging systems are part of numerous fertility clinics world-wide. We discuss the physics of the Hoffman imaging system from optical thickness to image intensity, implement a simple, yet fast, reconstruction algorithm using Fast Fourier Transformation and discuss the usability of the method on a number of cells from a human embryo. Novelty is identifying the non-linearity of a typical Hoffman imaging system, and the application of Fourier Transformation to reconstruct the optical thickness.
\end{abstract}

\section{Hoffman Modulation Contrast}

Hoffman Modulation Contrast (HMC) is a non-invasive light microscopy contrast technique for the study of so-called phase objects, which only affects the phase of the transmitted light. Because of its ability to generate contrast in images of otherwise transparent phase objects in a non-invasive manner, it is widely used for in vivo studies of biological specimen. Specifically, it is the favorite/standard technique used at many fertility clinics for the evaluation of embryo vitality. The wish for quality assessment in fertility treatments, has led to the study of computational methods for 3D object reconstruction and quantitative morphological measurements based on HMC images. This paper describes a method for reconstructing the optical thickness profile from a HMC microscopy image of a transparent but anomalous refracting object. The method uses a simple model of the HMC image formation isolating the nonlinearities, and it is applied to isolated human blastomeres in a study of their volumes. Previous and related work is path-length estimation in differential interference contrast images [1,2] and general regularization techniques [3,4].

The HMC technique generates contrast by converting "phase gradients" or "optical gradients" into intensity variations in microscopy images [5] such that positive and negative gradients appear bright and dark respectively, an example 


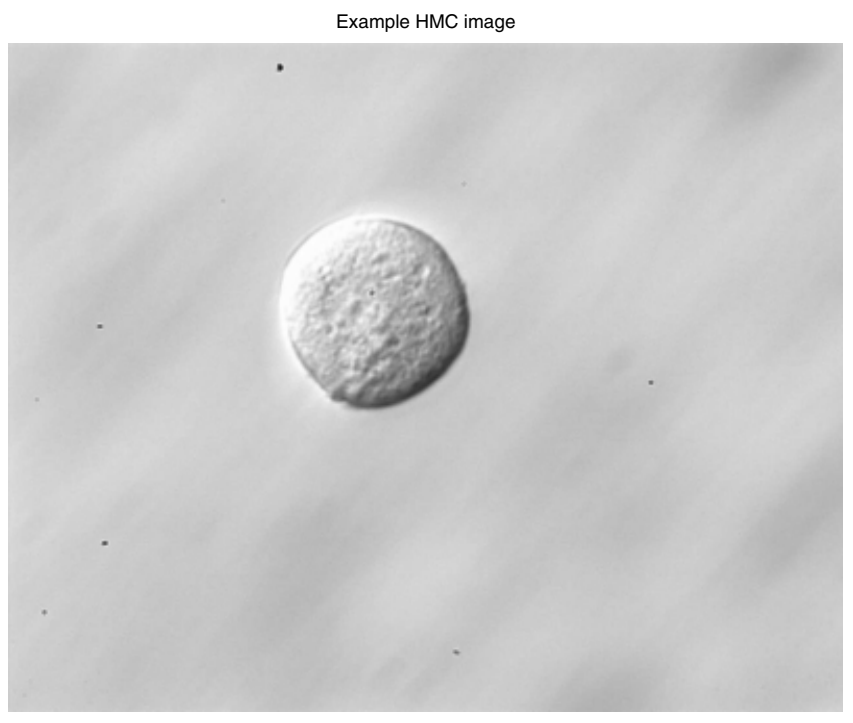

(a)

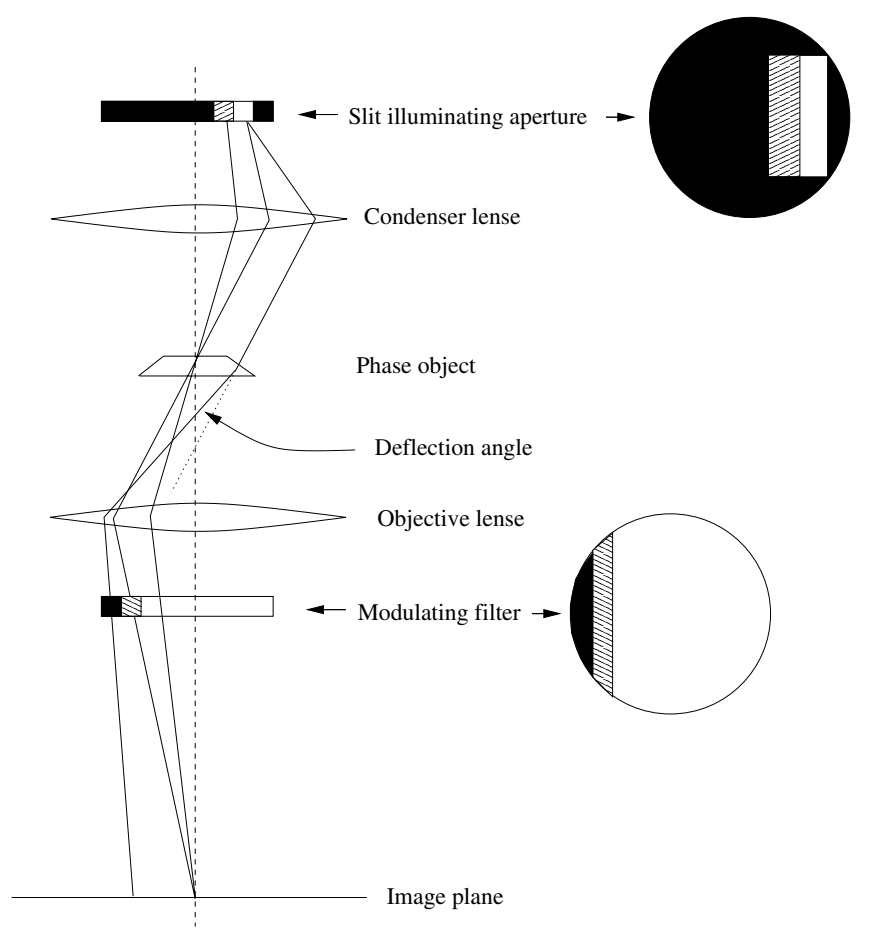

(b)

Fig. 1. (a) HMC image of a human blastomere, (b) The HMC Microscope model [6]. 
of which is shown in Fig. 1(a). This results in images that give a human observer the familiar but false impression [5] of looking at the highlights and shadows resulting from an illuminated surface with height variations. As illustrated in Fig. 1(b), the HMC microscope uses a rectangular slit illuminating aperture placed in the front focal plane of the condenser of the microscope, together with an aligned amplitude modulating filter with a dark, a gray, and a bright region placed in the conjugate back-focal plane of the objective lens [5,7]. In [6] this was refined using linear polarization filters giving control of the effective transmittance of a second slit placed next to the original slit.

\section{HMC Image Model}

To reconstruct the optical thickness using HMC, the original model from [7, Eq. 10-11] has been adopted to the refined technique described in [6]. In this case there are 3 intervals of local linearity shown in Fig. 2(a). The function

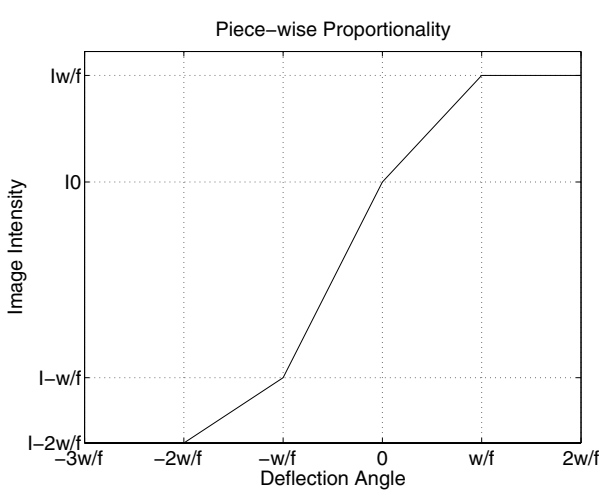

(a)
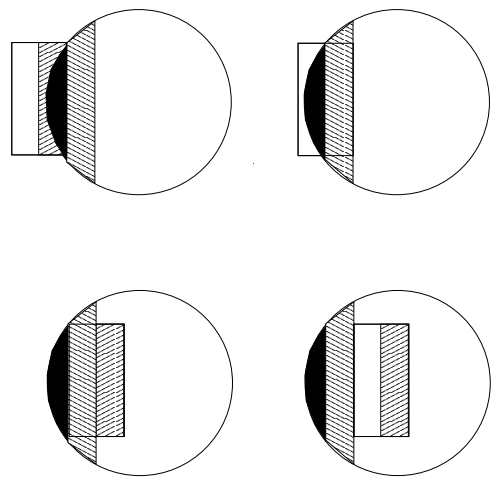

(b)

Fig. 2. Mapping deflection angles to intensities. (a) The image intensity as function of deflection angle in refined model. (b) The 4 images of the slits in the HMC modulating filter giving rise to 4 discontinuities.

has 4 first order discontinuities at deflection angles $-2 w / f,-w / f, 0$, and $w / f$ corresponding to the 4 positions of the image of the slits in the HMC modulating filter shown in Fig. 2(b). At the discontinuities the intensity may be found to be: $I_{-2 w / f}=I_{i} \alpha T_{D}+I_{i} T_{D}, I_{-w / f}=I_{i} \alpha T_{G}+I_{i} T_{D}, I_{0}=I_{i} \alpha T_{B}+I_{i} T_{G}$, and $I_{+w / f}=I_{i} \alpha T_{B}+I_{i} T_{B}$, where $f$ is the focal length of the microscope, $w$ is the width of the slits, $I_{i}$ is the incident intensity for each of the two slits, $\alpha$ is the transmittance of the semi-transparent slit, and $T_{B}, T_{G}$ and $T_{D}$ are the 
transmittances of respectively the bright, gray, and dark regions of the HMC modulating filter. Linear interpolation between these give:

$$
\begin{aligned}
& I\left(S_{\theta}\right)-I_{0}= \\
& \qquad \begin{array}{lc}
I_{i}(\ldots) ; & S_{\theta} \leq-2 \frac{w}{f} \\
I_{i}\left(\left(T_{G}-T_{D}\right)\left(\alpha\left(\frac{f}{w} S_{\theta}+1\right)-1\right)+\alpha\left(-T_{B}+T_{G}\right)\right) ; & -2 \frac{w}{f} \leq S_{\theta} \leq-\frac{w}{f} \\
I_{i}\left(\alpha\left(T_{B}-T_{G}\right)+\left(T_{G}-T_{D}\right)\right) \frac{f}{w} S_{\theta} ; & -\frac{w}{f} \leq S_{\theta} \leq 0 \\
I_{i}\left(T_{B}-T_{G}\right) \frac{f}{w} S_{\theta} ; & 0 \leq S_{\theta} \leq \frac{w}{f} \\
I_{i}(\ldots) ; & \frac{w}{f} \leq S_{\theta}
\end{array}
\end{aligned}
$$

Here $S_{\theta}=\cos \theta \frac{\partial}{\partial x} S+\sin \theta \frac{\partial}{\partial y} S$ is the directional derivative of the optical thickness $S$ in the slit width direction, which in terms of the magnitude of the gradient of the optical thickness $|\nabla S|$ is given by $S_{\theta}=|\nabla S| \cos \beta$, where $\beta$ is the angle between the gradient of the optical thickness and the slit width direction, $\theta$.

\section{Model Approximations}

A good model for the majority of living cells and tissues is that they are completely transparent with spatially varying speed of light $[8, \mathrm{p} .3]$, and that they may be modeled by the real-valued refractive index $n(x, y, z)=c_{s} / c$, where $c_{s}$ and $c$ are the speed of light of the surrounding medium and in the cell. We shall use a coordinate system with the $z$-axis along the optical axis, and assume that the object is located in a thin layer above the $x y$-plane $(z=0)$. The refraction experienced by a ray of light is assumed to be well approximated by anomalous diffraction [9, p. 115], which is a good approximation even for objects having large finite extend, $z_{0}$ in the sense $\frac{2 \pi}{\lambda} z_{0}>>1$, as long as they are soft, $|n-1|<<1$, and have $\frac{2 \pi}{\lambda}(n-1)$ approximately constant throughout the object.

In this model the object is represented by the finite phase delay, $\phi(x, y)=$ $\int_{0}^{z_{0}} \frac{2 \pi}{\lambda}(n(x, y, z)-1) d z$, which is the delay experienced by a ray traveling in the negative $z$-direction and intersecting the object plane at $(x, y, z=0)$, and where $\lambda$ is the wavelength of light in the surrounding medium. The optical path length difference or "optical thickness profile",

$$
\begin{aligned}
S(x, y) & =\frac{\lambda}{2 \pi} \phi(x, y) \\
& =\int_{0}^{2 \pi}(n(x, y, z)-1) d z,
\end{aligned}
$$

has the advantage over the phase delay, that it is independent of the wavelength or frequency of the light, and thus only depends on the object. This is illustrated for a homogeneous "sphere" in 2D in Figure 3

The approximate model of paraxial rays [10, p. 207] known as Gaussian Optics [10, p. 167] is adopted. Taking the $z$-axis as the optical axis of main ray propagation, i.e. for ray direction vector in polar coordinates $\boldsymbol{d}=\left(d_{x}, d_{y}, d_{z}\right)=$ $(\cos (\eta) \sin (\xi), \sin (\eta) \sin (\xi), \cos (\xi))$, the angle $\xi$ is assumed small enough such 


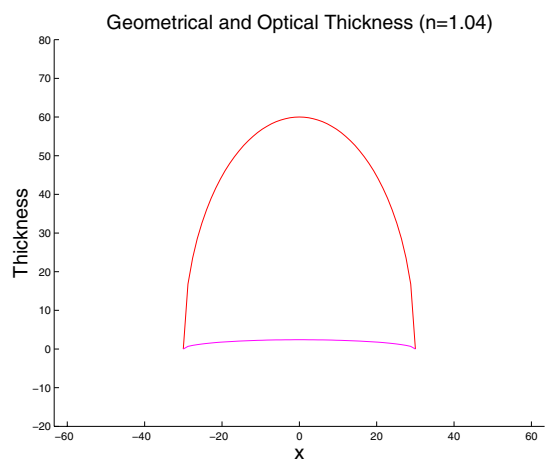

(a)

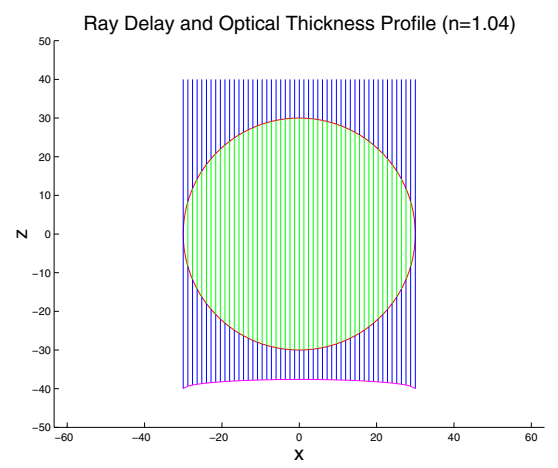

(b)

Fig. 3. (a) The geometrical thickness profile and the optical thickness profile for a homogeneous sphere. (b) Ray delays for the same optical thickness profile.

that $\sin (\xi) \simeq \xi$, and thus $\boldsymbol{d} \simeq(\cos (\eta) \xi, \sin (\eta) \xi, 1)$. Suppose a paraxial ray with direction vector $\boldsymbol{d}=\left(d_{x}, d_{y}, d_{z}\right)$ is incident on an anomalous diffracting object with constant "optical gradient" $\nabla S(x, y)=\left(k_{1}, k_{2}\right)$. The optical thickness profile is then given as $S(x, y)=k_{1} x+k_{2} y+S_{0}$. The corresponding phase delay profile for a monochromatic paraxial ray with wavelength $\lambda$ in the surrounding medium is then $\phi(x, y)=2 \pi / \lambda S(x, y)=2 \pi / \lambda\left(k_{1} x+k_{2} y+S_{0}\right)$. If the phase function of the incoming wave is written: $\phi_{i}(x, y, z)=2 \pi / \lambda\left(d_{x} x+d_{y} y+d_{z} z\right)$ we see that the refracted wave in the $(x, y)$-plane $(z=0)$ will have the phase function $\phi_{r}(x, y)=\phi_{i}(x, y, 0)+\phi(x, y)=\left(d_{x}+k_{1}\right) x+\left(d_{y}+k_{2}\right) y+S_{0}$, which we recognize as the phase function of a plane wave with direction cosines $\left(d_{x}+k_{1}, d_{y}+k_{2}\right)$.

Diffraction phenomena, arising from the finite aperture of the image forming objective lens, are not modeled. This is in contrast to the extensive theory of the resolution limits of image formation in diffraction limited systems, however, the objects and the resolution at which they are studied are assumed to be sufficiently larger than the wavelength of light. Human embryos have a diameter of approximately $120 \mu m=240 \lambda$.

\section{Automated, Fast and Regularized Reconstruction}

The HMC derivative direction may be estimated as the dominating direction of the structure tensor [11] at global scale. In the present algorithm a variant of the structure tensor is used [12], where the apparent direction of light is estimated as the largest eigenvector of the $2 \times 2$ matrix

$$
T_{i j}(x, y, \sigma)=\int_{\Omega} P(x, y, \sigma)\left(S_{\theta, x, \sigma} S_{\theta, y, \sigma}\right) d x d y
$$


with $P(x, y, \sigma)=k\left(S_{\theta, x, \sigma}^{2}+S_{\theta, y, \sigma}^{2}\right)$, where $* * \cdot$ is the convolution operator, $k$ is a normalizing constant such that $P(x, y, \sigma)$ integrates to unity, $S_{\theta, x, \sigma}=\frac{\partial}{\partial x} G_{\sigma} * S_{\theta}$, $S_{\theta, y, \sigma}=\frac{\partial}{\partial y} G_{\sigma} * S_{\theta}$, and $G_{\sigma}(x, y)=\left(2 \pi \sigma^{2}\right)^{-1} \exp \left(-\left(x^{2}+y^{2}\right) /\left(2 \sigma^{2}\right)\right)$.

Given the HMC derivative direction, $\theta$, the optical thickness in a noiseless image may be reconstructed using, $S(x, y)=\int_{-\infty}^{\left.l_{x, y, \theta}^{-1}(x, y)\right)} S_{\theta}\left(l_{x, y, \theta}(t)\right) d t$, where $l_{x, y, \theta}(t)$ is a line parameterized by $t$ passing through the point $(x, y)$ at an angle $\theta$ w.r.t. the image coordinate system, such that $l_{x, y, \theta}(0)=(x, y)$, and $S_{\theta}$ is the measured image. Unfortunately, noise in $S_{\theta}$ will have profound influence on the numerical stability of the reconstruction, so the integration is regularized by penalizing violent neighboring fluctuations. This is achieved by minimizing $\int_{\Omega} F\left(S_{\theta}(x, y), L_{x}(x, y), L_{y}(x, y)\right) d x d y$, where $L_{x}$ and $L_{y}$ are the derivatives of $L$ w.r.t the coordinate axis, $\Omega$ the image domain, $F\left(S_{\theta}, L_{x}, L_{y}\right)=\tau\left(L_{x}^{2}+L_{y}^{2}\right)+$ $\left(S_{\theta}-\cos (\theta) L_{x}-\sin (\theta) L_{y}\right)^{2}$, and $\tau$ is the regularization parameter. Arguments for $S_{\theta}, L_{x}$, and $L_{y}$ have been omitted for brevity. Using Euler-Lagrange it is found that the minimum must satisfy, $0=\partial_{x} F_{L_{x}}+\partial_{y} F_{L_{y}}$, leading to

$$
\tilde{L}=\frac{-2 \cos (\theta) i u-2 \sin (\theta) i v}{\left.2 \tau\left(u^{2}+v^{2}\right)+2 \cos ^{2}(\theta) u^{2}+2 \sin ^{(} \theta\right) v^{2}+4 \cos (\theta) \sin (\theta) u v} \tilde{S}_{\theta},
$$

where $\tilde{L}$ and $\tilde{S}_{\theta}$ are the Fourier Transform of the $L$ and $S_{\theta}$, and $(u, v)$ are the frequency directions corresponding to $(x, y)$.

\section{Evaluation and Conclusion}

We have reconstructed the optical thickness profile of 8 isolated human blastomeres using the method described above, and compared the results with the apparent geometrical volume, estimated from the $2 \mathrm{D}$ contour and assumption of spherical geometry. The images were acquired using the FertiGrab system from IH-Medical, Image House A/S, Denmark.

Based on simple measurements and calibration experiments we used the constants $T_{d}=0, T_{g}=0.5, T_{b}=1, w / f=0.19$, and $T_{2}=0.64$ in the conversion of image intensities to deflection angles by inversion of (1). The background intensity, $I_{0}$, is readily acquired in an image where the uniform non-refracting medium is visible. Further, experimenting with different values of the regularization parameter, $\tau$, lead us to set it as low as possible without resulting in images visibly disturbed by noise, $\tau=0.05$. We found that increasing $\tau$ after a certain threshold resulted in a systematic decrease in optical thickness. The Fourier implementation assumes periodic boundary condition (torus structure), and to amend, a spatial finite difference scheme was implemented with identical optimization functional but fixed, zero boundaries, in order to refine the initial estimate of the Fourier Method.

Using the reconstructed thickness profiles, we may estimate the diameter $\left(D_{z}\right)$ of the blastomere along the z-axis from the average relative refractive index, as: $S_{c}=D_{z}\left(n / n_{s}-1\right)$. In order to measure the "optical vertical diameter" we need to know $n / n_{s}-1$. Unfortunately, it has not been possible to 
find these data in the literature for human blastomeres. Based on our analysis of other measurements, we have come to believe that human blastomeres have an average relative refractive index to the surrounding water of approximately $n / n_{s}=1.38 / 1.33=1.04$. Calculation of the diameters along the z-axis of $8 \mathrm{hu}-$ man blastomeres in comparison with a mean estimate $D_{c}$ from the $2 \mathrm{D}$ contour is given in Table 5. It is also possible to estimate the "optical volume" of the

\begin{tabular}{|c|c|c|c|c|c|}
\hline Blastomere & $\begin{array}{r}D_{c} \\
/ \mu m\end{array}$ & $\begin{array}{r}S \max \\
/ \mu m\end{array}$ & $\begin{array}{r}D_{z} \\
/ \mu m\end{array}$ & $\begin{array}{c}\frac{4}{3} \pi\left(D_{c} / 2\right)^{3} \\
/(10 \mu m)^{3}\end{array}$ & $\begin{array}{r}V_{O} /\left(n / n_{s}-1\right) \\
/(10 \mu m)^{3}\end{array}$ \\
\hline 1 & 48 & 3.1 & 82 & 59 & 55 \\
\hline 2 & 52 & 2.5 & 66 & 72 & 28 \\
\hline 3 & 50 & 2.8 & 75 & 64 & 40 \\
\hline 4 & 49 & 2.8 & 75 & 63 & 38 \\
\hline 5 & 57 & 3.1 & 82 & 100 & 58 \\
\hline 6 & 50 & 2.5 & 65 & 64 & 31 \\
\hline 7 & 51 & 2.3 & 61 & 68 & 29 \\
\hline 8 & 51 & 1.9 & 52 & 69 & 32 \\
\hline
\end{tabular}

Table 1. Blastomere data.

object by integrating the optical thickness over the support of the object image, $V_{O}=\int_{\text {Object area }} S(x, y) d A=\int_{V}\left(n / n_{s}-1\right) d V$, where $V$ is the cylinder volume described by the object contour along the z-axis. The volumes have been computed for the 8 blastomeres (also presented in the table) scaled with $n / n_{s}-1$ for ease of comparison with the geometrical volume estimate. The geometrically and optically estimated volumes differ up to a factor of 2 . We must emphasize that ground truth is not available, and the deviations in the two models may be attributed misfits in both models. The blastomeres have been placed at random orientation, and it is observed that their images are close to circular. It thus seems implausible that the cells are elongated along the z-direction. Further, the balance between the internal an external forces on a blastomere cell in isolation will cause the cell membrane to approach spherical shape. Therefore spherical is the most likely cell shape. However, we note that the spherical model is clearly limited in its lack of parameters. For the optical thickness model, many of the parameters have only been estimated loosely, and we assume that a more careful calibration will increase the accuracy.

The presented algorithm is capable of retrieving $3 \mathrm{D}$ information in a noninvasive manner otherwise lost in studies of 2D HMC images. Future work will focus on improving the model to increase the accuracy of $3 \mathrm{D}$ information from the 2D HMC images especially in studying blastomeres in embryos.

\section{References}

1. C. Preza, E.B. van Munster, J.A. Aten, D.L. Snyder, and F.U. Rosenberger. Determination of direction-independent optical path-length distribution of cells using 
rotational-diversity transmitted-light differential interference contrast (dic) images. In C.J. Cogswell, J.A. Conchello, and T. Wilson, editors, Three-Dimensional Microscopy: Image Acquisition and Processing V, volume 3261 of Proceedings of SPIE, pages 60-70. SPIE, 1998.

2. D. Young, C.A. Glasbey, A.J. Gray, and N.J. Martin. Towards automatic cell identification in DIC microscopy. Journal of Microscopy, 192:186-193, 1998.

3. A.N. Thikonov and V.Y. Arseninn. Solution of ill-posed problems. V.H. Winston \& Sons, John Wiley, 1977.

4. M. Nielsen, L. Florack, and R. Deriche. Regularization, scale space, and edge detection filters. Journal on Mathematical Imaging and Vision, 7(4):291-307, 1997.

5. R. Hoffman and L. Gross. The modulation contrast microscope. Nature, 254:586588, April 1975.

6. R. Hoffman. The modulation contrast microscope: principles and performance. Journal of Microscopy, 110:205-222, August 1977.

7. R. Hoffman and L. Gross. Modulation contrast microscope. Applied Optics, 14(5):1169-1176, May 1975.

8. S. Bradbury. An Introduction to the Optical Microscope, volume 01 of Royal Microscopical Society MICROSCOPY HANDBOOKS. BIOS Scientific Publishers Ltd, 9 Newtec Place, Magdalen Road, Oxford OX4 1RE, UK, revised edition, 1989.

9. W.T. Grandy Jr. Scattering of Waves from Large Spheres. Cambridge University Press, 2000.

10. M. Born and E. Wolf. Principles of Optics, Electromagnetic Theory of Propagation, Interference and Diffraction of Light. Cambridge University Press, seventh (expanded) edition, 1999.

11. J. Weickert. Anisotropic diffusion in image processing. Teubner Verlag, Stuttgart, 1998.

12. X. Zabulis, J. Sporring, and S.C. Orphanoudakis. Scale summarized and focused browsing of primitive visual content. In Proceedings of the Fourth International Conference on Visual Information Systems, volume 1929 of Lecture Notes in Computer Science, pages 269-278, Lyon, France, 2000. Springer Verlag. 